\title{
Economic Model to Evaluate the Cost-Effectiveness of Second-Line Nilotinib Versus Dasatinib for the Treatment of Philadelphia Chromosome-Positive Chronic Myeloid Leukemia (CML-CP) in Italy
}

\author{
Massimiliano Bonifacio ${ }^{1}$. Vikalp Maheshwari ${ }^{2} \cdot$ Diana $\operatorname{Tran}^{3}$ [D $\cdot$ Gianluca Agostoni ${ }^{4} \cdot$ Kalitsa Filioussi $^{4}$. \\ Ricardo Viana ${ }^{5}$
}

Accepted: 24 June 2021 / Published online: 23 July 2021

(C) The Author(s) 2021

\begin{abstract}
Objective The aim of this study was to evaluate the cost effectiveness of second-line nilotinib versus dasatinib for the treatment of Philadelphia chromosome-positive chronic myeloid leukemia (CML-CP) patients who are intolerant or resistant to imatinib and can transition to treatment-free remission (TFR).

Methods A partitioned survival model was developed to compare the cost effectiveness of nilotinib versus dasatinib. The model was developed from the Italian healthcare payer perspective and included the following health states: on second-line tyrosine kinase inhibitor (TKI), off second-line TKI, accelerated phase/blastic crisis, TFR, and death. Progression-free and overall survival curves were derived from patient-level data that compared nilotinib and dasatinib as second-line therapy in CML-CP patients who were resistant or intolerant to imatinib. Drug costs, healthcare costs, and adverse event costs were based on real-world evidence and publicly available databases. Cost effectiveness was estimated over a 40-year time horizon. Scenario analyses were performed by adjusting time horizon, TFR parameters, costs, and utilities.

Results Second-line nilotinib resulted in greater time spent in TFR (0.91 life-years), increased quality-adjusted life-years (QALYs) (1.89), increased life-years (2.16), and decreased per-patient costs (-38,760 €). Therefore, nilotinib was strongly dominant compared with dasatinib in the base-case analysis. Nilotinib remained strongly dominant in most scenario analyses including shorter time horizon, exclusion of TFR, and varying TKI drug costs.

Conclusions While the model showed that nilotinib treatment of imatinib-intolerant or resistant CML-CP patients was more effective and less costly than dasatinib treatment, there is considerable uncertainty in the findings.
\end{abstract}

\section{Key Points for Decision Makers}

Based upon real-world comparative effectiveness data, nilotinib treatment of previously-treated CML patients was found to dominate dasatinib treatment, by increasing patient benefit at a reduced cost per patient. This

Ricardo Viana

ricardo.viana@novartis.com

1 Section of Hematology, Department of Medicine, University of Verona, Verona, Italy

2 Novartis Healthcare Pvt. Ltd., Hyderabad, India

3 EVERSANA Life Science Services, LLC, Burlington, Ontario, Canada finding stems from nilotinib inducing a greater molecular response, increasing patients' years in treatment-free remission (TFR), and having a lower cost of drug treatment.

Inclusion of TFR leads to greater cost savings and QALY gains and should be considered in future costeffectiveness analyses.

4 Region Europe Value and Access, Novartis Farma S.p.A, Origgio, Italy

5 Global Value \& Access, Oncology, Novartis Pharma AG, WSJ- Fabrikstrasse 18-3.330, 4056 Basel, Switzerland 


\section{Introduction}

Chronic myeloid leukemia (CML) is a myeloproliferative disorder which, if untreated, can lead to bleeding, infections, organ failure, and death [1]. Global CML incidence is $1-1.5$ per 100,000 people [1], with Italy having a standardized incidence of 1.16 and 1.40 per 100,000 adult women and men, respectively [2]. CML is characterized in most cases by the presence of the Philadelphia chromosome $(\mathrm{Ph})$ [1], wherein the chromosome 9 Abelson gene $(a b l)$ is fused with the chromosome 22 breakpoint cluster region gene ( $b c r)$ [3]. The $a b l$ gene expresses a tightly regulated tyrosine kinase responsible for cellular processes and critical to proper T-cell receptor signaling [4]. Transcription of the $b c r-a b l$ gene results in a constitutively active fusion protein with tyrosine kinase activity that can lead to increased proliferation, decreased apoptosis, and dysregulated adhesion [1].

Standard of care for CML is treatment with a tyrosine kinase inhibitor (TKI) (i.e., imatinib, nilotinib, dasatinib, bosutinib, or ponatinib) $[3,5]$. Tyrosine kinase inhibitors induce molecular response (i.e., reduction of $b c r-a b l$ transcript levels) and improve progression-free survival (PFS) and overall survival (OS) [3, 5]. Indeed, TKIs have markedly improved patient survival, with 5-year OS in imatinib- and nilotinib-treated patients estimated to be $91.7 \%$ and $93.7-96.2 \%$, respectively [6]. An emerging goal of TKI treatment of CML is achievement of treatment-free remission (TFR), wherein patients can discontinue therapy and maintain major molecular response [7]. Actually, the Gruppo Italiano Malattie EMatologiche dell'Adulto (GIMEMA; Italian Group for Hematologic Diseases of the Adult) CML Working Party (WP), in their recommendations for managing CML, recently recognized TFR as the main goal for virtually all patients, including those in the second-line setting [8]. Patients are eligible to attempt TFR after achieving a deep molecular response, which is defined as molecular response $4\left(\mathrm{MR}^{4}\right.$; BCR-ABL1 ${ }^{\mathrm{IS}}<$ $0.01 \%)$ or molecular response $4.5\left(\mathrm{MR}^{4.5}\right.$; BCR-ABL1 ${ }^{\mathrm{IS}}$ $\leq 0.0032 \%)[9,10]$. Once in TFR, approximately $40 \%$ of patients remain in TFR and those that relapse remain sensitive to TKIs [11].

With survival of CML patients approaching that of the general population, long-term TKI treatment can lead to budgetary pressures that could be alleviated through TFR and treatment discontinuation [12]. Indeed, recent budget impact analyses estimated that TFR could reduce total CML treatment costs in Italy by more than 54 million euros over 7 years [13], in Lebanon by more than $\$ 7$ million (USD) over 4 years [14], and in Japan by over $\$ 66$ million (USD) over 3 years [15]. Cost-effectiveness analyses (CEAs) are also now demonstrating the impact of TFR in increasing quality-adjusted life-years (QALYs) and decreasing costs in first-line treatment of patients with CML with TKIs $[16,17]$, yet consideration for TFR in CEAs of second-line treatment of CML has not been shown. Previous CEAs of second-line treatment for patients with CML who are resistant or intolerant to imatinib have compared second-line nilotinib and dasatinib [18-20] or second-line nilotinib, dasatinib, and high-dose imatinib [21-23]. These studies, however, have failed to consider TFR and therefore may underestimate the value of CML therapies approved for TFR [18-21].

The European Medicines Agency (EMA) has approved nilotinib (Tasigna ${ }^{\circledR}$ ) for use in newly diagnosed and previously imatinib-treated patients [24], but it is most commonly used after imatinib as a second-line option. Importantly, nilotinib is the only TKI approved, by the EMA, for physician-monitored TFR in select patients [24]. Dasatinib is another second-generation TKI recommended by the European Society for Medical Oncology (ESMO) clinical practice guidelines in cases of failure of first-line therapy (generally imatinib) and as an alternative to nilotinib [7]. The goal of this study was to assess the cost effectiveness of nilotinib in comparison with dasatinib in patients who are intolerant or resistant to imatinib, and can transition to TFR. The analysis was designed from the Italian healthcare payer perspective and was compliant with European LeukemiaNet (ELN) 2013 treatment switching recommendations [9].

\section{Methods}

\subsection{Model Overview}

No CEAs incorporating second-line TFR were identified; however, an existing model comparing the cost effectiveness of second-line nilotinib and dasatinib was adapted to include the ability to model TFR as a health state. Based on this previously published model [18], a partitioned survival model (Fig. 1) was developed that compared nilotinib with dasatinib in eligible CML patients who were previously treated with imatinib. A hypothetical cohort of 1000 previously treated adults with $\mathrm{Ph}+$ chromosome-positive chronic myeloid leukemia (CML-CP) requiring second-line nilotinib or dasatinib was modeled. A 40-year model duration was chosen to reflect a lifetime horizon, with 1-month cycles. The model assumed the perspective of the Italian healthcare payer, Servizio Sanitario Nazionale (National Health Service), and considered only costs related to healthcare resources. Half-cycle corrections and discounting (3\% to costs and effects, as per Italian guidelines for value dossier submissions issued by the Italian Medicines Agency) were applied [25]. The health outcomes for determining cost effectiveness were reported as life-years and QALYs. 


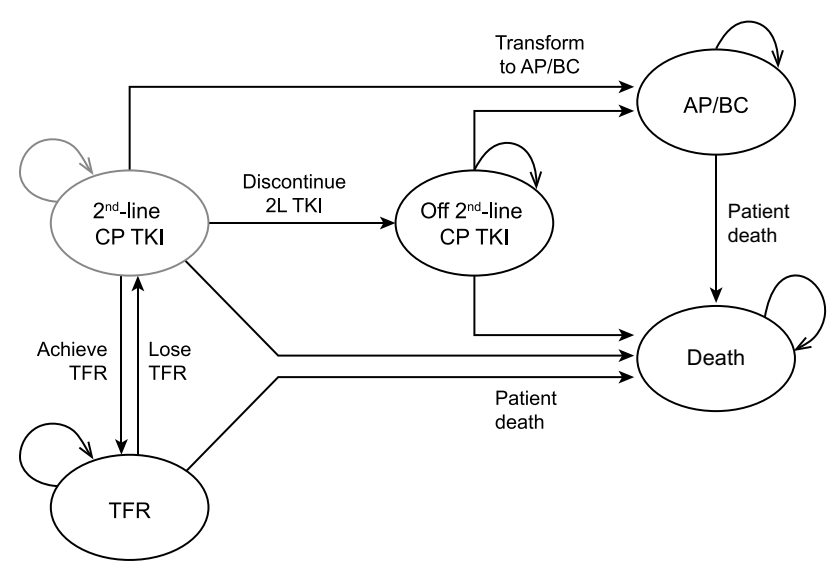

Fig. 1 Schematic of the cost-effectiveness model. $A P / B C$ accelerated phase/blastic crisis, $C P$ chromosome-positive, $T F R$ treatment-free remission, TKI tyrosine kinase inhibitor

Modeled patients could be in the following health states: 'second-line TKI', 'TFR', 'off second-line TKI', 'accelerated phase/blastic crisis (AP/BC)', or 'death' (Fig. 1). All patients enter the model on second-line TKI in chronic phase (i.e., 'second-line TKI'). Although the percentage of patients who could achieve and maintain $\mathrm{MR}^{4.5}$ was available from ENESTop, the estimate from this clinical trial might not reflect second-line TFR eligibility in reality [26]. Fifty percent of patients remaining on second-line therapy and achieving $\mathrm{MR}^{4.5}$ were assumed to maintain their deep molecular response and could transition to the TFR health state after 12 months, based on a more reasonable estimate from ENESTcmr [27]. Patients on nilotinib and dasatinib were both able to transition to TFR (based on ENESTop trial for nilotinib and the DASFREE trial for dasatinib) [26, 28]. Patients in TFR that relapsed returned to the chronic phase and did not become TFR eligible again. In line with the 2013 ELN clinical guidelines for the management of CML [9], patients could transition from the 'second-line TKI' health state to the 'off second-line TKI' health state if they had an inadequate major cytogenic response (MCyR) by 12 months to their second-line TKI. These patients would start thirdline therapy (assumed to be bosutinib or ponatinib). Any patient on TKI treatment (but not in TFR) could transition to the AP/BC health state due to disease progression. Patients could die due to all-cause mortality while in any health state.

\subsection{Model Inputs}

As described previously, an existing model [18] used PFS and OS curves derived from patient-level data from a United States retrospective chart review that compared nilotinib and dasatinib as second-line therapy in $\mathrm{Ph}+\mathrm{CML}-\mathrm{CP}$ patients who were resistant or intolerant to imatinib [29]. The extrapolated exponential curves were digitized and included in the

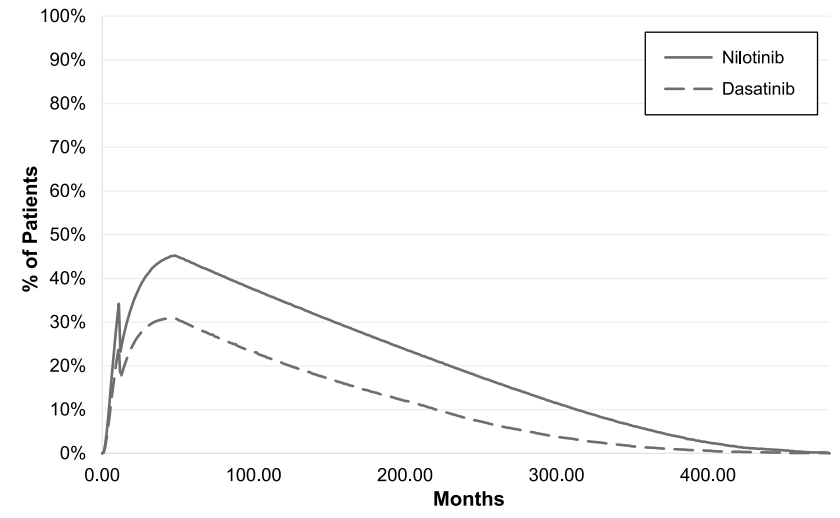

Fig. 2 Proportion of patients with MR4.5 in model (calculated in model). MR4.5 molecular response 4.5

present model as the only available data source for estimates of long-term comparative effectiveness. To determine eligibility to transition to the TFR health state in our model, rates of $\mathrm{MR}^{4.5}$ over time for nilotinib were obtained from the ENESTcmr trial, a 48-month, open-label, randomized phase III study that assessed deep molecular response rates in patients receiving second-line nilotinib [30]. Rates of $\mathrm{MR}^{4.5}$ were not available for dasatinib and thus were estimated through application of a hazard ratio (1.37) to the ENESTcmr data (Fig. 2) [31]. The hazard ratio was derived from comparative evidence from a real-world chart review of 280 second-line CML patients that found nilotinib treatment to be associated with a significantly higher rate of achieving $\mathrm{MR}^{4.5}$ than dasatinib [31]. Duration of second-line TFR was based on digitized TFR curves from the discontinuation trials for nilotinib (ENESTop) and for dasatinib (DASFREE) [26, 28]. Prior to 12 months, the time on treatment (TOT) curve followed the PFS curve. After 12 months, the TOT curve was assumed to decrease at the rate of the OS curves specific to each treatment arm. The population starting age

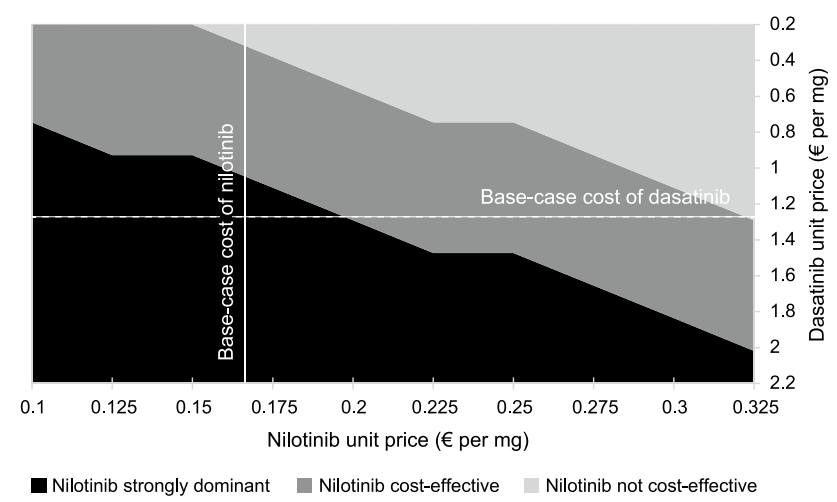

Fig. 3 Two-way sensitivity analysis varying the cost of dasatinib and nilotinib. Cost effectiveness is based on a willingness-to-pay threshold of $87,330 €$ 
was set to 60 years old and $65 \%$ of the hypothetical cohort was male based on source data patient characteristics $[18$, 29].

The average health utility of the Italian general population is 0.92 [32]. Patients in TFR were assumed to also have a health state utility of 0.92 , as these patients approached a 'functional cure'. The utility of the remaining health states in the model were determined by applying a disutility derived from data by respondents in the United Kingdom according to Szabo et al. [33], a multinational time trade-off preference survey conducted among patients with CML in Australia, the United Kingdom, Canada, and the United States. Disutilities were 0.09 for second-line TKI and 0.22 for AP/BC. The disutility value for discontinued second-line TKI was assumed to be the same as second-line TKI (Table 1).

The model included CML-related drug costs, healthcare resource costs (including monitoring and resource utilization), adverse event (AE)-related costs, and end-of-life costs reflecting €2020 values.

The daily dose of nilotinib and dasatinib were assumed to be $598.2 \mathrm{mg}$ and $122.7 \mathrm{mg}$, respectively, based upon an analysis of real-world dosage [31]. Monthly drug costs for TKIs were $3040.02 €$ for nilotinib and $4748.73 €$ for dasatinib [34]. The monthly drug cost for discontinued second-line TKI (regardless of prior treatment) was $4490.42 €$, which was calculated by weighting common third-line TKI treatment, based upon real-world estimates of use of nilotinib
(35\%), dasatinib (40\%), bosutinib (14\%), or ponatinib (12\%) [35]. Patients in TFR received no monthly drug acquisition costs, while patients in $\mathrm{AP} / \mathrm{BC}$ were assumed to have drug acquisition costs of $6142.11 €(50 \%$ ponatinib and $50 \%$ bosutinib).

Costs of BCR-ABL monitoring and hematologist visits were included, with resource use patterns considered by health state. Hematologist visits were assumed to occur monthly in all health states at a fee of $21.22 € /$ visit [36]. BCR-ABL monitoring (208.49 €/test) was assumed to occur once every 3 months in all health states, except TFR, where it was assumed to occur monthly [7, 13, 36].

Rates of AEs were obtained from a real-world chart review of second-line TKIs conducted by Novartis (Supplementary Table 1, see Electronic Supplementary Material [ESM]) [37]. Severe or life-threatening AEs (grade 3 and 4) were included in the model. It was assumed that the realworld evidence values reflect 24 -month rates. Adverse event rates were converted to a per-cycle probability in the model and applied to patients on second-line TKIs in each cycle up to month 24. The AE management cost per AE experienced was derived from hospitalization tariffs [38]. The assumption that each AE required hospital resources is plausible given the severity of AEs included in the model, and is the same assumption used in an Italian CEA of ponatinib in CML [36]. Based on the costs and the frequencies of AEs for both treatments, nilotinib had a slightly lower calculated
Table 1 Summary of model inputs

\begin{tabular}{ll}
\hline Model parameter & Reference case value \\
\hline Perspective & Italian healthcare payer \\
Number of CML-CP patients & 1000 \\
Time horizon of analysis & 40 years \\
Cycle length & 1 month \\
Molecular response criteria for TFR eligibility & 4.5 \\
Cost of molecular monitoring & $208.49 €$ \\
Cost of hematologist visit & $21.22 €$ \\
Cost of molecular monitoring and physician visits (per month) & \\
Second-line TKI, off second-line TKI CP, AP/BC & $90.71 €$ \\
TFR & $229.70 €$ \\
Drug costs (per month) & \\
Nilotinib (second-line TKI CP) & $3040.02 €$ \\
Dasatinib (second-line TKI CP) & $4748.73 €$ \\
Off second-line TKI CP (nilotinib, dasatinib, bosutinib, ponatinib) & $4490.42 €$ \\
AP/BC (bosutinib + ponatinib) & $6142.11 €$ \\
Health state utility decrements & \\
Second-line TKI and off second-line TKI CP & 0.09 \\
AP/BC & 0.22 \\
\hline
\end{tabular}

$A P / B C$ accelerated phase/blastic crisis, $C M L$ chronic myeloid leukemia, $C P$ chromosome-positive, $M R^{4.5}$ molecular response 4.5, TFR treatment-free remission, TKI tyrosine kinase inhibitor

${ }^{a}$ Utility decrements were applied to the assumed baseline utility of 0.92 for the Italian general population. Patients in TFR received no decrement 
per-patient cost of managing grade 3 and 4 AEs than dasatinib (5.32€ vs $7.92 €)$.

End-of-life costs were assumed to be $8010.81 €$, which is in line with the estimate for cost of death due to disease from previous Italian economic analyses for TKIs in CML $[13,36]$.

\subsection{Scenario Analyses}

The following scenario analyses were run to assess the robustness of the cost-effectiveness result to plausible or pessimistic alternative inputs: same $\mathrm{MR}^{4.5}$ rate for dasatinib and nilotinib (hazard ratio 1.0), variable percentage of patients assumed to maintain sustained $\mathrm{MR}^{4.5}$ for 12 months (43.6\% and 77.3\%, based on ENESTcmr and ENESTop, respectively $[26,30])$, a shorter time horizon (10 years, consistent with the time horizon used by previous CEAs considering TFR $[16,17])$, different discount rates ( $0 \%$ and $5 \%$ [25]), reduced cost of dasatinib (by 50\%), increased cost of nilotinib (by 50\%), exclusion of AE costs, exclusion of AP/BC drugs costs, exclusion of end-of-life costs, exclusion of TFR, and variation in the TFR and general population utilities ( 0.85 and 1 as used in recent CML CEAs incorporating TKI discontinuation [16, 17], and utility of 0.85 for the TFR health state and utility of 0.92 for general population). A two-way sensitivity analysis, where the costs of dasatinib and nilotinib were varied simultaneously, was also conducted to identify the prices at which nilotinib would remain cost effective against dasatinib.

\section{Results}

\subsection{Base Case}

The base case investigated the cost effectiveness of nilotinib compared with dasatinib for the treatment of previously
treated-CML patients. Second-line nilotinib was found to be strongly dominant over dasatinib (Table 2). Treatment with nilotinib, over a lifetime horizon, was associated with greater patient benefits including greater time spent in TFR (0.91 life-years), increased QALYs (1.89), increased life-years (2.16), and less time spent in advanced disease ( -0.16 lifeyears). Treatment with nilotinib also decreased per-patient costs $(-38,760 €)$. Per-patient cost savings mostly arose due to the additional nilotinib patients that transitioned to, and stayed within, TFR, as well as the lower monthly drug cost, compared with dasatinib.

\subsection{Scenario Analyses}

Nilotinib remained cost saving and more effective in most scenario analyses including shorter time horizon, alternative discount rates, varying the percentage of patients eligible for TFR, exclusion of TFR, and varying TKI drug costs and utilities (Table 3). Shortening the time horizon to 10 years further increased cost savings to an estimated $-63,898 €$ due to the shorter treatment duration for incurring nilotinib drug cost. With a longer time horizon, the higher OS associated with nilotinib therapy would result in more patients alive and receiving therapy in the nilotinib arm compared with the dasatinib arm, thereby decreasing the incremental costs as in the base case. Exclusion of TFR and reducing the number of patients who could transition to TFR were associated with increased costs and decreased QALYs for both dasatinib and nilotinib as the drug cost-offset and higher utility afforded by patients being in the TFR state were not considered. Although TFR was a driver of decreased incremental costs for second-line nilotinib, exclusion of TFR still resulted in lower incremental costs $(-35,108 €)$ and higher QALYs gained (1.81) for nilotinib. Similarly, nilotinib was still associated with lower incremental costs $(-10,941 €)$ and higher incremental QALYs (1.85) even when dasatinib had the same rate of $\mathrm{MR}^{4.5}$ for TFR eligibility. Scenarios

Table 2 Discounted lifetime per-patient results by health state

\begin{tabular}{|c|c|c|c|c|c|c|c|c|c|}
\hline & \multicolumn{3}{|l|}{ Dasatinib } & \multicolumn{3}{|l|}{ Nilotinib } & \multicolumn{3}{|c|}{ Incremental } \\
\hline & Costs $(€)$ & LYs & QALYs & Costs $(€)$ & LYs & QALYs & Costs $(€)$ & LYs & QALYs \\
\hline On 2L TKI & 283,192 & 4.874 & 4.045 & 196,715 & 5.234 & 4.344 & $-86,477$ & 0.360 & 0.298 \\
\hline On TFR & 3746 & 1.359 & 1.250 & 6242 & 2.265 & 2.083 & 2496 & 0.905 & 0.833 \\
\hline Off 2L TKI & 87,128 & 1.585 & 1.315 & 144,867 & 2.635 & 2.187 & 57,739 & 1.050 & 0.872 \\
\hline $\mathrm{AP} / \mathrm{BC}$ & 128,646 & 1.720 & 1.204 & 116,637 & 1.559 & 1.092 & $-12,009$ & -0.161 & -0.112 \\
\hline Dead & 5748 & & & 5239 & & & -509 & & \\
\hline Total & 508,460 & 9.538 & 7.815 & 469,700 & 11.693 & 9.706 & $-38,760$ & 2.155 & 1.891 \\
\hline ICER & & & & & & & $\begin{array}{r}\text { Nilotinib } \\
\text { QALYs }\end{array}$ & $\begin{array}{l}\text { (2.155) an } \\
\text { dasatinib }\end{array}$ & \\
\hline
\end{tabular}

$2 L$ second-line, $A P / B C$ accelerated phase/blastic crisis, ICER incremental cost-effectiveness ratio, $L Y$ life year, $Q A L Y$ quality-adjusted life-year, $T F R$ treatment-free remission, $T K I$ tyrosine kinase inhibitor 
Table 3 Scenario analysis results

\begin{tabular}{|c|c|c|c|c|}
\hline Variable & Test value & $\begin{array}{l}\text { Incremental } \\
\text { costs }(€)\end{array}$ & $\begin{array}{l}\text { Incremental } \\
\text { QALYs }\end{array}$ & Cost per QALY Gained $(€)^{\mathrm{a}}$ \\
\hline Base case & & $-38,760$ & 1.89 & Strongly dominant \\
\hline Time horizon & 10 years & $-63,898$ & 0.63 & Strongly dominant \\
\hline \multirow[t]{2}{*}{ Discount rate (costs \& effects) } & $0 \%$ & $-21,011$ & 2.99 & Strongly dominant \\
\hline & $5 \%$ & $-43,896$ & 1.45 & Strongly dominant \\
\hline Dasatinib cost reduced & $50 \%$ & 88,276 & 1.89 & 46,687 \\
\hline Nilotinib cost increased & $50 \%$ & 63,372 & 1.89 & 33,516 \\
\hline Dasatinib $\mathrm{MR}^{4.5}$ (hazard ratio with nilotinib) & 1.0 & $-10,941$ & 1.85 & Strongly dominant \\
\hline \multirow[t]{2}{*}{ Percentage of patients eligible for TFR } & $43.6 \%$ & $-37,472$ & 1.88 & Strongly dominant \\
\hline & $77.3 \%$ & $-45,883$ & 1.94 & Strongly dominant \\
\hline \multirow[t]{3}{*}{ Utility of TFR and general population } & 0.85 & $-38,760$ & 1.74 & Strongly dominant \\
\hline & 1.0 & $-38,760$ & 2.06 & Strongly dominant \\
\hline & 0.85 for TFR only & $-38,760$ & 1.83 & Strongly dominant \\
\hline No TFR & & $-35,108$ & 1.81 & Strongly dominant \\
\hline No adverse event costs & & $-38,714$ & 1.89 & Strongly dominant \\
\hline No AP/BC drug costs & & $-26,926$ & 1.89 & Strongly dominant \\
\hline No end-of-life costs & & $-38,251$ & 1.89 & Strongly dominant \\
\hline
\end{tabular}

$A E$ adverse event, $A P / B C$ accelerated phase/blastic crisis, $M R$ molecular response, $Q A L Y$ quality-adjusted life-year $T F R$ treatment free remission

a 'Strongly dominant' indicates that nilotinib was associated with lower costs and higher QALYs than dasatinib

varying discounting rates, excluding costs, or varying the utility value of being in TFR also did not change the conclusions of the base-case analysis. However, decreasing the unit cost of dasatinib by $50 \%$ and increasing the unit cost of nilotinib by $50 \%$ led to scenarios with added costs (incremental cost-effectiveness ratios [ICERs] in the northeast quadrant), suggesting that the analysis is sensitive to TKI drug costs.

The results of the two-way sensitivity analysis varying the unit costs of nilotinib and dasatinib are shown in Fig. 3. Nilotinib remained the strongly dominant or costeffective strategy in most areas when comparing the ICERs at an informal willingness-to-pay threshold of 87,330 $€$ per QALY for oncology drugs in Italy [39]. Nilotinib was the more preferred treatment strategy in tested scenarios with dasatinib cost reductions of up to $84 \%$ and nilotinib cost increases up to $195 \%$.

\section{Discussion}

This study assessed the cost effectiveness of nilotinib compared with dasatinib in the second-line treatment setting from the perspective of the Italian healthcare payer (National Health Service), and was compliant with switching recommendations in the ELN 2013 guidelines and the emerging treatment goal of TFR in the Italian GIMEMA CML WP recommendations $[8,9]$. To our knowledge, this model is the first to report on the cost effectiveness of second-line TKIs in CML considering TFR. Our model demonstrated nilotinib treatment of CML patients to strongly dominate (i.e., increased benefits and decreased costs) dasatinib therapy. The inclusion of TFR increased the estimated cost savings of nilotinib, emphasizing the value of nilotinib as the only marketed TKI with an approved product label for physician-monitored TFR in select patients. Importantly, nilotinib was found to be strongly dominant despite having attributed benefits of TFR to dasatinib, which does not have an approved TFR indication to support this.

The base-case results showed that over a 40 -year time horizon, treatment with nilotinib rather than dasatinib led to a change of 2.16 life-years, 1.89 QALYs, and a decreased cost per patient $(-38,760 €)$. Compared with dasatinib, treatment with nilotinib, over a lifetime horizon, was associated with greater time spent in TFR ( 0.91 life-years) based on estimated $\mathrm{MR}^{4.5}$ responses (Fig. 2). Similar to the current analysis, Li et al. [18] found that in comparison with dasatinib, nilotinib was associated with better health outcomes (by 2.2 life-years and 1.9 QALYs) and lower total costs (by US $\$ 12,655)$. In contrast, the current analysis found greater cost savings ( $-38,760 €$ vs - US $\$ 12,655)$ than Li et al. [18], likely due to the inclusion of TFR.

The scenario analysis where the percentage of patients eligible for TFR (due to maintenance of $\mathrm{MR}^{4.5}$ response) was greater than the base case (i.e., 77.3\%) further decreased incremental costs and increased incremental QALYs for nilotinib. Actually, in our analyses, nilotinib was strongly dominant or cost effective in all scenarios, despite dasatinib-treated patients also benefiting from TFR. Nilotinib 
remained strongly dominant in the scenario that completely excluded TFR from the model.

Consideration of TFR can be important for calculating costs and QALYs in CEAs in CML [16, 17], yet other comparable CEAs of second- and subsequent-line TKIs did not consider TFR in their models, and also often lacked mature data and/or real-world data needed to accurately model longterm effectiveness in a real-world setting [18-23]. These past CEAs have reported nilotinib to be more cost effective than dasatinib, similarly cost effective to dasatinib (in comparison with high-dose imatinib), or inferior to dasatinib [18, 21-23]. The exclusion of TFR in previous studies neglects a key difference in the treatment benefits for nilotinib versus dasatinib, namely that nilotinib is associated with a significantly higher rate of achieving $\mathrm{MR}^{4.5}$ than dasatinib, resulting in a greater proportion of patients on nilotinib being able to transition to TFR [30, 31]. Early studies have been limited by the lack of long-term follow-up of randomized or single-arm trials and observational studies used to inform model inputs such as OS and PFS [19, 21, 22], as well as studies with long-term follow-up to inform durability of molecular response rates [22]. Generally, results that found nilotinib to be inferior or similar to dasatinib utilized relatively immature clinical effectiveness data that were subject to large degrees of uncertainty. This limited conclusions that could be made around estimating life-years and QALYs used to compare cost-effectiveness between treatments. Previous CEAs also lacked detailed information on treatment adherence that might be observed outside of a clinical trial setting $[18,19,21]$. This lack of treatment adherence data led to assumptions of $100 \%$ adherence, which may have led to an overestimation of treatment costs and overlooked potential differences in dosing between treatments. Our study uses data that has become available related to deep molecular response rates needed for TFR eligibility [30], comparative evidence on patient outcomes between TKIs from a realworld chart review [31], and time in TFR [26, 28]. In studies using more mature and real-world data, such as the study by Li et al. [18], nilotinib has been found to be cost effective, if not strongly dominant, compared with dasatinib. The combination of mature data, real-world estimates of dosing and $\mathrm{MR}^{4.5}$ rates [40], and inclusion of TFR in this study led to the estimation that nilotinib strongly dominated dasatinib.

A major limitation of this study is a lack of head-to-head data comparing the efficacy of nilotinib and dasatinib and their respective molecular response rates. The best estimate of the comparative effectiveness for PFS and OS between nilotinib and dasatinib as second-line therapies in patients with CML-CP was found to be a real-world, retrospective medical chart review completed by Griffin et al. [29]. Furthermore, this chart review was completed in the United States rather than Italy. The best relative estimate of $\mathrm{MR}^{4.5}$ rates between nilotinib and dasatinib was also obtained from a real-world chart review study by Cortes et al. [31]. A retrospective review of adult CML patients treated at Italian hematologic centers also compared rates of deep molecular response between those who received second-line dasatinib $(n=95)$ versus nilotinib $(n=68)$ [41]. In contrast to Cortes et al. [31], the authors found that deep molecular response at $\mathrm{MR}^{4}$ was not significantly different between patients who received dasatinib versus nilotinib. Given that the treatment guidelines and product label recommend a deep molecular response threshold of $\mathrm{MR}^{4.5}$, the data from the Cortes et al. [31] real-world study was used in the present analysis, while the same $\mathrm{MR}^{4.5}$ rate was used for nilotinib and dasatinib in a scenario analysis (hazard ratio 1). Importantly, even with the assumption of the scenario analysis, where nilotinib and dasatinib produce similar rates of $\mathrm{MR}^{4.5}$, nilotinib remains strongly dominant.

Another limitation of this study is the lack of a probabilistic sensitivity analysis (PSA). The previously published model [18] that this economic evaluation was based on did not report a PSA. Given the lack of variance information for key efficacy parameters, the conjoint uncertainty surrounding all the parameter estimates that populated the model was not fully assessed.

This study also had several assumptions that should be acknowledged. First, similar to Li et al. [18], the same health state cost is applied for patients who discontinued their second-line TKI, regardless of whether their second-line TKI was nilotinib or dasatinib. Second, for patients who achieve $\mathrm{MR}^{4.5}$ (based on Kaplan-Meier curve from ENESTcmr), the model assumes $50 \%$ will maintain their $\mathrm{MR}^{4.5}$ for a full 12 months and be eligible to attempt TFR (i.e., $50 \%$ of patients will lose $\mathrm{MR}^{4.5}$ response prior to TFR). This assumption was based upon data from ENESTcmr, which demonstrated that $43.6 \%$ of patients maintained $\mathrm{MR}^{4.5}$ for an additional year after at least 2 years of second-line nilotinib treatment; as no data exists for second-line dasatinib $\mathrm{MR}^{4.5}$ maintenance, assuming the same rate as nilotinib was deemed reasonable [27]. Data from ENESTop found that a much higher percentage of patients (77.3\%) would maintain $\mathrm{MR}^{4.5}$ for a year [26]. Therefore, assuming a 50\% rate of eligibility to attempt TFR was considered to be a realistic and conservative estimate, as scenario analyses showed greater cost savings with higher rates of $\mathrm{MR}^{4.5}$ maintenance. Third, the model assumes that $100 \%$ of patients eligible for TFR will choose to attempt TFR, which may not reflect real-world practice. Lastly, the TFR curve (i.e., the number of patients in TFR) is dependent on the $\mathrm{MR}^{4.5}$ curve (which is based on the TOT curve). The TOT curve is based on the PFS and OS curves, which were derived from Li et al. [18]. To avoid curves crossing due to use of different datasets, we assumed a hierarchy of OS $>$ PFS $>$ TOT $>\mathrm{MR}^{4.5}>$ TFR at any timepoint, such that the TFR curve will become the $\mathrm{MR}^{4.5}$ curve when they meet. 
Despite these limitations and assumptions, it is important to stress that the model reflects evidence-based clinical decisions surrounding TFR as a treatment goal. Transitions through the model health states were informed by treatment recommendations from the ELN 2013 guidelines and realworld data, thus demonstrating the economic benefits of assuming guideline adherence in clinical practice.

\section{Conclusions}

Based upon real-world comparative effectiveness data and the consideration for TFR, treatment of previously treated CML patients in the Italian setting with nilotinib was found to strongly dominate dasatinib treatment, by increasing patient benefit at a reduced cost per patient. This finding stems from nilotinib inducing a greater molecular response, more patient-years on TFR, and a lower cost of drug treatment. Nilotinib also remained cost effective in all scenario analyses with alternative model assumptions and the majority of the two-way sensitivity analysis examining lower unit costs of dasatinib, due to the efficacy and associated TFR benefits of nilotinib. As the new treatment goal for patients with CML, TFR is an effective cost-reduction strategy that warrants inclusion in any analyses comparing economic benefits of interventions. Future CEAs of subsequent-line treatment of patients with CML with TKIs should similarly consider TFR to avoid overestimating costs and underestimating QALYs for treatments indicated for TFR. Furthermore, the use of real-world evidence should be a preferred alternative data source in the absence of direct head-to-head clinical trial data. Where possible, future studies should utilize real-world effectiveness data to derive 'real-life' economic conclusions that would be generalizable to patients and healthcare providers.

Supplementary Information The online version contains supplementary material available at https://doi.org/10.1007/s41669-021-00286-3.

Acknowledgements The authors thank Teige Bourke of EVERSANA for providing medical writing support.

\section{Declarations}

Funding Support for the conduct and reporting of this study was provided by Novartis Pharma AG.

Conflicts of interest/Competing interests The following authors are employed by the sponsor: Vikalp Maheshwari, Gianluca Agostoni, Kalitsa Filioussi, and Ricardo Viana. Massimiliano Bonifacio receives personal fees and non-financial support from the sponsor outside the submitted work. Diana Tran is an employee of EVERSANA, which received funding from Novartis Pharmaceuticals to develop the model described in this manuscript.
Ethics approval Not applicable.

Consent to participate Not applicable.

Availability of data and material The data, model, and methodology used in the research are proprietary.

Code availability The programming code in the model used in the research is proprietary.

Authors' contributions VM, DT, and RV contributed to the concept and design. VM and GA contributed to the acquisition of data. MB, $\mathrm{VM}, \mathrm{GA}, \mathrm{KF}$, and RV contributed to the analysis and interpretation of data. MB, VM, DT, GA, KF, and RV contributed to the drafting and review of the manuscript. RV contributed to the funding and supervision of the study.

Open Access This article is licensed under a Creative Commons Attribution-NonCommercial 4.0 International License, which permits any non-commercial use, sharing, adaptation, distribution and reproduction in any medium or format, as long as you give appropriate credit to the original author(s) and the source, provide a link to the Creative Commons licence, and indicate if changes were made. The images or other third party material in this article are included in the article's Creative Commons licence, unless indicated otherwise in a credit line to the material. If material is not included in the article's Creative Commons licence and your intended use is not permitted by statutory regulation or exceeds the permitted use, you will need to obtain permission directly from the copyright holder. To view a copy of this licence, visit http://creativecommons.org/licenses/by-nc/4.0/.

\section{References}

1. Emole J, Talabi T, Pinilla-Ibarz J. Update on the management of Philadelphia chromosome positive chronic myelogenous leukemia: role of nilotinib. Biologics. 2016;10:23-31. https://doi.org/ 10.2147/BTT.S67844.

2. Hoffmann V, Baccarani M, Hasford J, Lindörfer D, Burgstaller S, Sertic D, et al. The EUTOS population-based registry: incidence and clinical characteristics of $2904 \mathrm{CML}$ patients in 20 European Countries. Leukemia. 2015;29(6):1336-43.

3. Pinilla-Ibarz J, Sweet KL, Corrales-Yepez GM, Komrokji RS. Role of tyrosine-kinase inhibitors in myeloproliferative neoplasms: comparative lessons learned. Onco Targets Ther. 2016;9:4937-57. https://doi.org/10.2147/ott.S102504.

4. Gu JJ, Ryu JR, Pendergast AM. Abl tyrosine kinases in T-cell signaling. Immunol Rev. 2009;228(1):170-83.

5. Larson RA. Is there a best TKI for chronic phase CML? Blood. 2015;126(21):2370-5. https://doi.org/10.1182/ blood-2015-06-641043.

6. Hochhaus A, Saglio G, Hughes TP, Larson R, Kim D, Issaragrisil S, et al. Long-term benefits and risks of frontline nilotinib vs imatinib for chronic myeloid leukemia in chronic phase: 5-year update of the randomized ENESTnd trial. Leukemia. 2016;30(5): 1044

7. Hochhaus A, Saussele S, Rosti G, Mahon F-X, Janssen JJ, HjorthHansen H, et al. Chronic myeloid leukaemia: ESMO Clinical Practice Guidelines for diagnosis, treatment and follow-up. Ann Oncol. 2017;28(4):41-51.

8. Baccarani M, Abruzzese E, Accurso V, Albano F, Annunziata $\mathrm{M}$, Barulli S, et al. Managing chronic myeloid leukemia for treatment-free remission: a proposal from the GIMEMA CML WP. Blood Adv. 2019;3(24):4280-90. 
9. Baccarani M, Deininger MW, Rosti G, Hochhaus A, Soverini S, Apperley JF, et al. European LeukemiaNet recommendations for the management of chronic myeloid leukemia: 2013. Blood. 2013;122(6):872-84. https://doi.org/10.1182/ blood-2013-05-501569.

10. National Comprehensive Cancer Network (NCCN). NCCN Clinical Practice Guidelines in Oncology (NCCN Guidelines): chronic myelogenous leukemia. Version 1. 2016. https://www.nccn.org/ professionals/physician_gls/pdf/cml.pdf. Accessed 2 Jun 2016.

11. Saußele S, Richter J, Hochhaus A, Mahon FX. The concept of treatment-free remission in chronic myeloid leukemia. Leukemia. 2016;30:1638. https://doi.org/10.1038/leu.2016.115.

12. Bower H, Björkholm M, Dickman PW, Höglund M, Lambert PC, Andersson TM-L. Life expectancy of patients with chronic myeloid leukemia approaches the life expectancy of the general population. J Clin Oncol. 2016;34(24):2851-2587.

13. Mennini F, Marcellusi A, Viti R, Saglio G. Budget Impact analysis of the first-line treatment of Philadelphia chromosome-positive chronic myeloid leukemia (Ph+ CML) adult patients. Farmecon Health Econ Therap Pathw. 2017;18(1):21-30.

14. Elias F, Gebran A, Said C, Beker RV, Ammar W. budget impact of treatment-free remission in treating chronic-phase philadelphiapositive chronic myeloid leukemia in Lebanon. J Glob Oncol. 2019;5:1-7.

15. Yamazaki K, Inagaki N, Moldaver D, Viana R, Kimura S. Budget impact analysis of treatment-free remission in nilotinibtreated Japanese chronic myeloid leukemia patients. Cancer Sci. 2020;111(7):2526.

16. Shih Y-CT, Cortes JE, Kantarjian HM. Treatment value of second-generation BCR-ABL1 tyrosine kinase inhibitors compared with imatinib to achieve treatment-free remission in patients with chronic myeloid leukaemia: a modelling study. Lancet Haematol. 2019;6(8):e398-e408. https://doi.org/10.1016/S2352-3026(19) 30087-0

17. Yamamoto C, Nakashima H, Ikeda T, Kawaguchi S-I, Toda Y, Ito $\mathrm{S}$, et al. Analysis of the cost-effectiveness of treatment strategies for CML with incorporation of treatment discontinuation. Blood Adv. 2019;3(21):3266-77.

18. Li N, Yang X, Fan L, Totev T, Guerin A, Chen L, et al. Nilotinib versus dasatinib as second-line therapy in patients with Philadelphia chromosome-positive chronic myeloid leukemia in chronic phase who are resistant or intolerant to imatinib: a cost-effectiveness analysis based on real-world data. J Med Econ. 2017;20(4):328-36. https://doi.org/10.1080/13696998.2016. 1261032.

19. Rogers G, Hoyle M, Thompson-Coon J, Moxham T, Liu Z, Pitt M et al. Dasatinib and nilotinib for imatinib-resistant or-intolerant chronic myeloid leukaemia: a systematic review and economic evaluation. Health Technol Assess. 2012;16(22):1-410. https:// doi.org/10.3310/hta16220

20. Whalen J, Stillman I, Ambavane A, Felber E, Makenbaeva D, Bolinder B. Cost-effectiveness analysis of second-line tyrosine kinase inhibitor treatment for chronic myelogenous leukemia. J Med Econ. 2016;19(5):445-61.

21. Loveman E, Cooper K, Bryant J, Colquitt JL, Frampton GK, Clegg A. Dasatinib, high-dose imatinib and nilotinib for the treatment of imatinib-resistant chronic myeloid leukaemia: a systematic review and economic evaluation. NIHR Health Technology Assessment programme: Executive Summaries. NIHR J Libr. 2012;16(23):iiixiii, 1-137. https://doi.org/10.3310/hta16230

22. Hoyle M, Rogers G, Moxham T, Liu Z, Stein K. Cost-effectiveness of dasatinib and nilotinib for imatinib-resistant or -intolerant chronic phase chronic myeloid leukemia. Value Health. 2011;14(8):1057-67. https://doi.org/10.1016/j.jval.2011.07.006.

23. Kulpeng W, Sompitak S, Jootar S, Chansung K, Teerawattananon Y. Cost-utility analysis of dasatinib and nilotinib in patients with chronic myeloid leukemia refractory to first-line treatment with imatinib in Thailand. Clin Ther. 2014;36(4):534-43. https://doi.org/10.1016/j.clinthera.2014.02.008.

24. European Medicines Agency. Human medicine European public assessment report (EPAR): Tasigna. https://www.ema.europa. eu/en/medicines/human/EPAR/tasigna. Accessed 20 Aug 2019.

25. Italian Medicines Agency. Linea guida per la compilazione del Dossier a supporto della domanda di rimborsabilità e prezzo di un medicinale. 2019. https://www.aifa.gov.it/en/linea-guidacapitolo-9. Accessed 1 Feb 2021.

26. Hughes TP, Boquimpani C, Takahashi N, Benyamini N, Clementino NCD, Shuvaev V, et al. ENESTop 144-Week Update: Long-Term Treatment-Free Remission (TFR) in Patients with Chronic Myeloid Leukemia in Chronic Phase (CML-CP) After Stopping Second-Line Nilotinib. Clin Lymphoma Myeloma Leuk. 2018;18:S222. https://doi.org/10.1016/j.clml.2018.07. 092.

27. Hughes TP, Cervantes F, Spector N, Leber B, Branford S, Glynos TA, et al. Treatment-free remission (TFR) eligibility in patients (pts) with chronic myeloid leukemia in chronic phase (CML-CP) and residual disease on long-term imatinib (IM) who switched to second-line nilotinib (NIL). DC: American Society of Hematology Washington; 2015.

28. Shah NP, García Gutiérrez JV, Jiménez-Velasco A, Larson SM, Saussele S, Rea D, et al. Dasatinib discontinuation in patients (Pts) with chronic-phase chronic myeloid leukemia (CML-CP) and stable deep molecular response (DASFREE). Blood. 2017;130(Suppl 1):314.

29. Griffin JD, Guerin A, Chen L, Macalalad AR, Luo J, Ionescu-Ittu $\mathrm{R}$, et al. Comparing nilotinib with dasatinib as second-line therapies in patients with chronic myelogenous leukemia resistant or intolerant to imatinib1a retrospective chart review analysis. Curr Med Res Opin. 2013;29(6):623-31. https://doi.org/10.1185/03007 995.2013.789012.

30. Hughes TP, Leber B, Cervantes F, Spector N, Pasquini R, Clementino NCD, et al. Sustained deep molecular responses in patients switched to nilotinib due to persistent BCR-ABL1 on imatinib: final ENESTcmr randomized trial results. Leukemia. 2017;31(11):2529-31. https://doi.org/10.1038/leu.2017.247.

31. Cortes J, Huynh L, Mendelson E, Brandt P, Dalal D, DerSarkissian $\mathrm{M}$, et al. Treatment patterns and deep molecular response in chronic phase-chronic myeloid leukemia patients treated with second-line nilotinib or dasatinib: a multi-country retrospective chart review study. Leuk Lymphoma. 2020;61(1):98-107.

32. Scalone L, Cortesi PA, Ciampichini R, Belisari A, D’Angiolella LS, Cesana G, et al. Italian population-based values of EQ-5D health states. Value Health. 2013;16(5):814-22.

33. Szabo SM, Levy AR, Davis C, Holyoake TL, Cortes J. A multinational study of health state preference values associated with chronic myelogenous leukemia. Value Health. 2010;13(1):103-11.

34. Servizio Sanitario Regione Autonoma della Sardegna ATS- Area Socio Sanitaria Locale Olbia. Proposal n.27 / 2017. http://www. aslolbia.it/documenti/2_47_20170126121213.pdf. Accessed 16 Jan 2020.

35. Kantar Health. CancerMPact ${ }^{\circledR}$, Patient Metrics. www.cance rmpact.com. Accessed 4 May 2020.

36. Lucioni C, Iannazzo S, Mazzi S. Valutazione di costo-efficacia di ponatinib nella terapia della leucemia mieloide cronica in Italia. Glob Reg Health Technol Assess. 2015;2:1-16.

37. Novartis. Data on File: Study Protocol Number MACS3668. 2018.

38. GAZZETTA UFFICIALE. Supplemento ordinario n. 8. 2013. https://www.trovanorme.salute.gov.it/norme/renderPdf.spring? seriegu $=$ SG\&datagu $=28 / 01 / 2013 \&$ redaz $=13$ A00528 $\&$ artp $=1 \&$ art $=1 \&$ subart $=1 \&$ subart $1=10 \&$ vers $=1 \&$ prog $=001$. Accessed 8 Feb 2021. 
39. Martone N, Lucioni C, Mazzi S, Fadda V. Valutazione di costoefficacia dei nuovi farmaci oncologici immessi sul mercato italiano. Glob Reg Health Technol Assess. 2014;1(2):31-43.

40. Cortes JE, Huynh L, Mendelson E, Brandt P, Dalal D, Dersarkissian M, et al. Treatment characteristics and deep molecular response in chronic phase-chronic myeloid leukemia patients treated with second-line nilotinib or dasatinib: a multi-country retrospective chart review Study. Blood. 2017;130(Suppl 1):2897.

41. Tiribelli M, Bonifacio M, Binotto G, Iurlo A, Cibien F, Maino E, et al. Excellent outcomes of 2G-TKI therapy after imatinib failure in chronic phase CML patients. Oncotarget. 2018;9(18):14219. 